ARTICLE

https://doi.org/10.1038/s41467-019-10906-6

\title{
Optogenetic control of Bacillus subtilis gene expression
}

Sebastian M. Castillo-Hair (10 1, Elliot A. Baerman², Masaya Fujita ${ }^{3}$, Oleg A. Igoshin (1) 1,2,4 \& Jeffrey J. Tabor (iD) 1,2

The Gram-positive bacterium Bacillus subtilis exhibits complex spatial and temporal gene expression signals. Although optogenetic tools are ideal for studying such processes, none has been engineered for this organism. Here, we port a cyanobacterial light sensor pathway comprising the green/red photoreversible two-component system CcaSR, two metabolic enzymes for production of the chromophore phycocyanobilin (PCB), and an output promoter to control transcription of a gene of interest into $B$. subtilis. Following an initial non-functional design, we optimize expression of pathway genes, enhance PCB production via a translational fusion of the biosynthetic enzymes, engineer a strong chimeric output promoter, and increase dynamic range with a miniaturized photosensor kinase. Our final design exhibits over 70 -fold activation and rapid response dynamics, making it well-suited to studying a wide range of gene regulatory processes. In addition, the synthetic biology methods we develop to port this pathway should make B. subtilis easier to engineer in the future.

\footnotetext{
${ }^{1}$ Department of Bioengineering, Rice University, 6100 Main St., Houston, TX 77005, USA. ${ }^{2}$ Department of Biosciences, Rice University, 6100 Main St., Houston, TX 77005, USA. ${ }^{3}$ Department of Biology and Biochemistry, University of Houston, 4800 Calhoun Rd., Houston, TX 77004, USA. ${ }^{4}$ Center for Theoretical Biophysics, Rice University, 6100 Main St., Houston, TX 77005, USA. Correspondence and requests for materials should be addressed to J.J.T. (email: jeff.tabor@rice.edu)
} 
$B$ acillus subtilis is a model organism for studying how time-varying (dynamic), heterogeneous, and spatiallycoordinated gene expression signals control single- and multicellular behaviors ${ }^{1-4}$. For instance, in response to short-term energy stress (i.e., glucose/phosphate limitation), transcription of the general stress response regulon is activated in a pulsatile manner with a frequency proportional to the stress intensity ${ }^{5}$. In contrast, environmental (e.g., osmotic) stress induces a single transcriptional pulse of this same regulon with an amplitude proportional to the rate of onset of the stress ${ }^{6}$. In a separate pathway, persistent starvation induces the master regulator of sporulation via a series of pulses of increasing amplitude ${ }^{7-9}$. Though all cells exhibit these pulses, only a subset go on to produce spores, possibly due to heterogeneity in levels of the master regulator ${ }^{10}$. Furthermore, prior to sporulation commitment, an excitable and noisy genetic circuit drives a small fraction of cells to transiently differentiate into a state competent for DNA uptake ${ }^{11-13}$. From a spatial perspective, coordinated gene expression patterns can be observed in biofilms of undomesticated B. subtilis, where subpopulations of motile, matrixproducing, and sporulating cells localize to different regions ${ }^{14}$. Furthermore, in these biofilms, metabolism and growth are synchronized across cells via potassium-mediated action potentials that radiate outwards from the center ${ }^{15,16}$.

Despite the richness of these regulatory dynamics, the underlying genetic circuits are typically studied using static and spatially homogenous genetic perturbations, including genetic knockouts $^{17}$ or the use of chemically-inducible promoters to express genes of interest at various steady state levels ${ }^{18,19}$. The inability to program artificial gene regulatory signals with precise temporal and spatial features in B. subtilis has limited understanding of even the most intensely-studied pathways. In contrast, engineered light-sensing two-component systems (TCS) have enabled exceptional control of gene expression dynamics in E. $\operatorname{coli}^{20}$, even at the single cell level ${ }^{21}$. Similar tools that enable precision control of $B$. subtilis gene expression are needed ${ }^{22}$.

The Synechocystis PCC6803 TCS CcaSR is a green light-activated/red light de-activated transcriptional regulatory pathway ${ }^{23}$ (Fig. 1). CcaSR comprises the cyanobacteriochrome (CBCR)family sensor kinase (SK) CcaS, and the OmpR/PhoB-family response regulator (RR) CcaR. CcaS contains a putative $\mathrm{N}$ terminal transmembrane helix, followed by a cGMP phosphodiesterase/adenylyl cyclase/FhlA (GAF) domain, two Per-ARNTSim (PAS) domains of unknown function, and a histidine kinase (HK) domain. CcaS senses light via the linear tetrapyrrole chromophore phycocyanobilin (PCB), a prosthetic group covalently bound to the GAF domain. PCB is synthesized in two steps: heme oxygenase 1 (encoded by ho1) oxidizes heme to biliverdin IX $\alpha$ (BV), and phycocyanobilin:ferredoxin oxidoreductase (encoded by $p c y A$ ) reduces $\mathrm{BV}$ to $\mathrm{PCB}$ (Fig. 1a) ${ }^{24,25}$. Holo-CcaS (hereafter $\mathrm{CcaS})$ is produced in a green light $(535 \mathrm{~nm})$-sensitive ground state with low autokinase activity. Absorption of a green photon switches CcaS to a red light $(672 \mathrm{~nm})$-sensitive active state with high autokinase activity. Active CcaS transfers a phosphoryl group from ATP to a conserved HK histidine residue (Fig. 1b), and subsequently to a conserved aspartate residue on $\mathrm{CcaR}$ (Fig. 1c). Phosphorylation activates CcaR, which subsequently induces transcription from the $\mathrm{P}_{c p c G 2}$ output promoter (Fig. 1c, d). Red light exposure reverts active CcaS to the ground state and de-activates $\mathrm{P}_{c p c G 2}$ transcription, likely through CcaS-mediated dephosphorylation of $\mathrm{CcaR}^{26}$ (Fig. 1b).

We and others have repurposed the CcaSR system as an optogenetic tool, and utilized it to achieve exceptional quantitative, spatial, and temporal control of gene expression in E. coli. In the original study, we cloned the unmodified $c c a S R$ genomic locus into an E. coli plasmid, which we co-transformed with a PCB production plasmid encoding a synthetic hol-pcyA operon ${ }^{27}$. However, this v1.0 system exhibits leaky output in red light and is activated less than 5 -fold by green. In a follow-up study, we decreased leakiness and increased dynamic range to over 100-fold by systematically optimizing expression of ccaS, ccaR, and the ho1-pcyA operon, and truncating $\mathrm{P}_{c p c G 2}$ to remove an unwanted transcriptional start site (resulting in $\left.\mathrm{P}_{c p c G 2-172}\right)^{28}$. Sode and coworkers later constructed several miniaturized CcaS variants lacking the PAS domains, and demonstrated that two of these proteins result in lower $\mathrm{P}_{c p c G 2}$ output in red light and similar or greater $\mathrm{P}_{c p c G 2}$ output in green ${ }^{29}$. We introduced the corresponding CcaS PAS deletions in the context of our optimized (v2.0) system, resulting in an E. coli CcaSR v3.0 system with very low leakiness and nearly 600-fold dynamic range ${ }^{30}$. Various versions of the CcaSR system have been used alone and in combination with additional optogenetic tools with different wavelength specificities to achieve precise spatial 27,31 and temporal $^{20,32,33}$ control of the expression of one or multiple genes, including at the single-cell level ${ }^{21}$. In one of the studies, we programmed linear ramps and sine waves of expression of a transcriptional repressor in order to characterize the input/output (I/O) dynamics of a widely-used synthetic gene circuit ${ }^{20}$.

Here, we combine lessons learned in our previous work with several novel synthetic biology approaches to port CcaSR into $B$. subtilis. Our initial design, which is based on E. coli CcaSR v2.0, does not respond to light. We utilize fluorescent protein fusions to reveal that hol, $p c y A$, and $c c a S$ are poorly expressed. Recoding of the initial ORF sequences and several modifications of the gene expression cassettes substantially improve expression. Despite these optimizations, we find that PCB levels remain low. Inspired by previous work on enzyme fusion ${ }^{34}$ and scaffolding ${ }^{35}$, we engineer a ho1-pcyA translational fusion, which results in high PCB levels. Next, we demonstrate that $\mathrm{P}_{c p c G 2-172}$, which is derived from Synechocystis PCC6803, is weak relative to other B. subtilis promoters. To increase transcriptional output, we chimerize $\mathrm{P}_{c p c G 2-172}$ with a strong constitutive $B$. subtilis promoter. Then, we increase CcaSR dynamic range by screening the best-performing miniaturized CcaS variants in the context of our system. Finally, we characterize the steady state and dynamic I/O of the optimized system, named B. subtilis CcaSR v1.0, to demonstrate that it should enable characterization of a wide range of $B$. subtilis gene regulatory processes. The principles elucidated during this debugging and optimization process should be of great utility to future $B$. subtilis synthetic biology applications, and more generally, to any situation where porting genetic circuits between bacterial species is required.

\section{Results}

B. subtilis CcaSR v0.1 does not respond to light. Our first implementation, named CcaSR v0.1, comprised three independent modules integrated into distinct genomic loci: the PCB production module (PPM v0.1, Fig. 1a), light-sensing module (LSM v0.1, Fig. 1b), and transcriptional output module (TOM v0.1, Fig. 1c). This design was based on the insights obtained from our previously engineered E. coli CcaSR circuit, where dynamic range increased with higher expression of hol and $p c y A$ and was optimal at intermediate $c c a S$ and $c c a R$ expression levels ${ }^{28}$. Thus, we engineered a synthetic hol-pcyA operon under control of the strong constitutive $B$. subtilis promoter $\mathrm{P}_{r p s D}{ }^{36}$ and separate copies of the synthetic ribosome binding site (RBS) MF001 (see "Methods" section) in PPM v0.1. Additionally, we expressed ccaS from the xylose-inducible promoter $\mathrm{P}_{x y l A}$ and its associated RBS ${ }^{18}$ and $c c a R$ from the IPTG-inducible promoter $\mathrm{P}_{\text {hy-spank }}{ }^{19}$ and synthetic RBS MF002 (see "Methods" section) in LSM v0.1 and TOM v0.1, respectively. Finally, to measure the output of the 


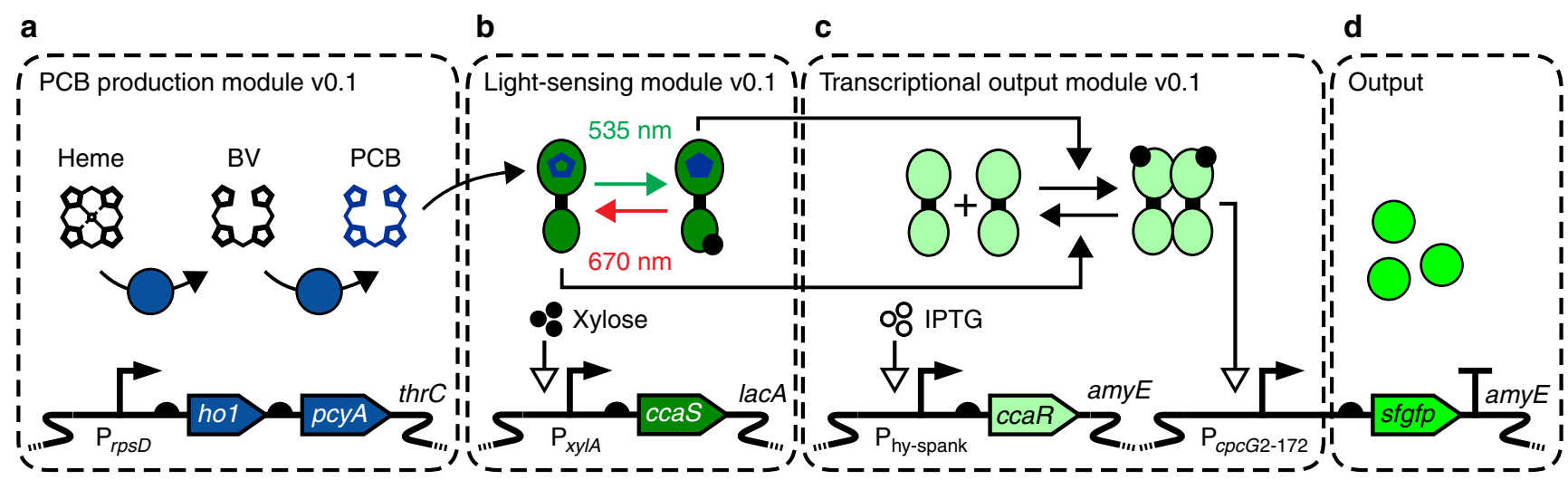

Fig. 1 B. subtilis CcaSR v0.1 device schematic. The first implementation of $B$. subtilis CcaSR, comprised of a PCB production module v0.1, b light-sensing module v0.1, and c transcriptional output module v0.1. d System activity is measured by an sfgfp reporter. Genetic diagrams are shown at the bottom, and proteins, prosthetic groups, and expected interactions are represented at the top

system, we encoded superfolder green fluorescent protein $(s f g f p)$ under $\mathrm{P}_{c p c G 2-172}$ and RBS MF001.

To characterize B. subtilis CcaSR v0.1, we induced expression of $c c a S$ and $c c a R$ by adding different combinations of xylose and IPTG concentrations under both red and green light, resulting in a total of 96 different conditions. Then, we assayed the output of the system by measuring the resulting sfGFP fluorescence in calibrated Molecules of Equivalent Fluorescein (MEFL) units via flow cytometry (see "Methods" section). We observed that even in the absence of xylose, sfGFP levels increased up to 40 -fold in response to IPTG addition (Supplementary Fig. 1B, C), likely due to CcaR phosphorylation from a small molecule donor such as acetyl phosphate ${ }^{37}$, while reporter activity was not detectable with a control TOM where the DNA binding domain of CcaR was removed (Supplementary Fig. 2). These results indicated that $\mathrm{CcaR}$ was properly expressed and capable of binding to and activating transcription from $\mathrm{P}_{c p c G 2-172}$ in $B$. subtilis. However, $B$. subtilis CcaSR v0.1 did not show any meaningful response to light under any of the conditions tested (Supplementary Fig. 1D). Furthermore, in contrast to E. coli CcaSR v2.028, addition of the ccaS inducer had little effect on transcription from $\mathrm{P}_{c p c G 2-172}$ (Supplementary Fig. 1B, C). Taken together, these results suggested that PCB was poorly produced, ccaS was poorly expressed, and/or CcaS was non-functional.

Debugging and optimizing PCB production. $\mathrm{Cph} 1(\mathrm{Y} 176 \mathrm{H})$ is an engineered Synechocystis PCC6803 phytochrome protein that becomes weakly red fluorescent upon PCB-binding ${ }^{38,39}$. We set out to utilize this protein to examine PCB levels produced from PPM v0.1 (Fig. 1a). To ensure that we could detect the fluorescent signal, we expressed $\operatorname{cph} 1(\mathrm{Y} 176 \mathrm{H})$ from our recent LacI-T7 promoter system, which results in very strong IPTG-inducible protein expression ${ }^{40}$. As a positive control, we added purified PCB (see "Methods" section) to B. subtilis cells overexpressing $\operatorname{cph} 1(\mathrm{Y} 176 \mathrm{H})$ with saturating IPTG. Surprisingly, the bacteria were very weakly red fluorescent $(15.7 \pm 1.9$ Molecules of Equivalent Allophycocyanin, MEAP) (Supplementary Fig. 3A, B) (see "Methods" section). We suspected that $\operatorname{cph} 1(\mathrm{Y} 176 \mathrm{H})$ was poorly expressed. To examine this possibility, we constructed a $c p h 1(Y 176 H)-s f g f p$ translational fusion and measured green fluorescence as before. Indeed, saturating IPTG yielded only $113 \pm 27$ MEFL of green fluorescence (Supplementary Fig. 3C, D) compared to $432,000 \pm 20,000$ MEFL when $s f g f p$ is expressed directly from the same LacI-T7 system ${ }^{40}$.
Highly expressed bacterial genes tend to exhibit little mRNA secondary structure near the $\mathrm{RBS}^{41-44}$. Computational analysis revealed that our synthetic RBS MF001 formed stable secondary structure with the initial $\operatorname{cph} 1(\mathrm{Y} 176 \mathrm{H})$ ORF sequence (Supplementary Fig. 3E). To increase expression, we replaced several of the first 15 codons with synonymous versions (resulting in $\left.\operatorname{cph} 1(\mathrm{Y} 176 \mathrm{H})^{\star}\right)$ predicted to reduce the problematic structure (Supplementary Fig. 3F). Indeed, this recoding process yielded a nearly 200-fold increase in IPTG-induced Cph1(Y176H)-sfGFP levels $(20,100 \pm 1,600$ MEFL, Supplementary Fig. 3D). As expected, induction of $\operatorname{cph} 1(\mathrm{Y} 176 \mathrm{H})^{\star}$ resulted in much stronger red fluorescence $(848 \pm 52 \mathrm{MEAP})$ in the presence of exogenously supplied PCB (Supplementary Fig. 3B).

We next integrated PPM v0.1 into our $\operatorname{cph} 1(\mathrm{Y} 176 \mathrm{H})^{*}$ strain, and measured red fluorescence to assay PCB production (Fig. 2, see "Methods" section). Unexpectedly, fluorescence remained below our limit of detection (Fig. 2). As with $\operatorname{cph} 1(Y 176 H)$, we suspected that hol and/or $p c y A$ may be poorly expressed leading to low PCB levels. Indeed, $s f g f p$ translational fusions to these two enzymes resulted in low fluorescence values $(246 \pm 14 \mathrm{MEFL}$ Ho1-sfGFP, 7.1 \pm 1.6 MEFL PcyA-sfGFP) (Supplementary Figs. 4, 5) compared to a control strain where only $s f g f p$ was expressed from $\mathrm{P}_{r p s D}(1580 \pm 190 \mathrm{MEFL})$ (Supplementary Fig. 6). Thus, we redesigned their expression cassette by separating both genes into independent transcriptional units, codon-optimizing the initial 15 codons of hol (resulting in $h o 1^{\star}$ ) and the complete sequence of $p c y A$ (resulting in $p c y A^{* *}$ ), and introducing a transcriptional terminator downstream of $p c y A^{* *}$ to eliminate potential mRNA instability ${ }^{45}$. These changes resulted in a dramatic increase in expression of both enzymes (21,400 \pm 1500 MEFL Hol-sfGFP, $8350 \pm 400$ MEFL for PcyA-sfGFP) (Supplementary Figs. 4, 5). Thus, a new PPM v0.2 design was constructed based on these modifications (Fig. 2). However, PPM v0.2 produced very little red fluorescence $(25.7 \pm 6.2 \mathrm{MEAP})$ via the $\operatorname{cph} 1(\mathrm{Y} 176 \mathrm{H})^{\star} \mathrm{PCB}$ probe. This result suggested that high levels of Hol and PcyA were not sufficient for robust PCB production in B. subtilis.

Enzyme colocalization has been shown to increase yield in synthetic metabolic pathways, likely by generating high local concentrations of intermediate metabolites that overcome thermodynamically unfavorable steps ${ }^{34,35}$. To test whether colocalizing Hol and PcyA could increase PCB yields, we constructed PPM v0.3, wherein $h o 1^{*}$ is translationally fused to $p c y A^{* *}$ via a flexible linker, and the fusion is again transcribed from $\mathrm{P}_{r p s D}$ (see "Methods" section). Strikingly, fusing these enzymes resulted in an 80 -fold increase in red fluorescence (2070 \pm 130 MEAP, Fig. 2), 


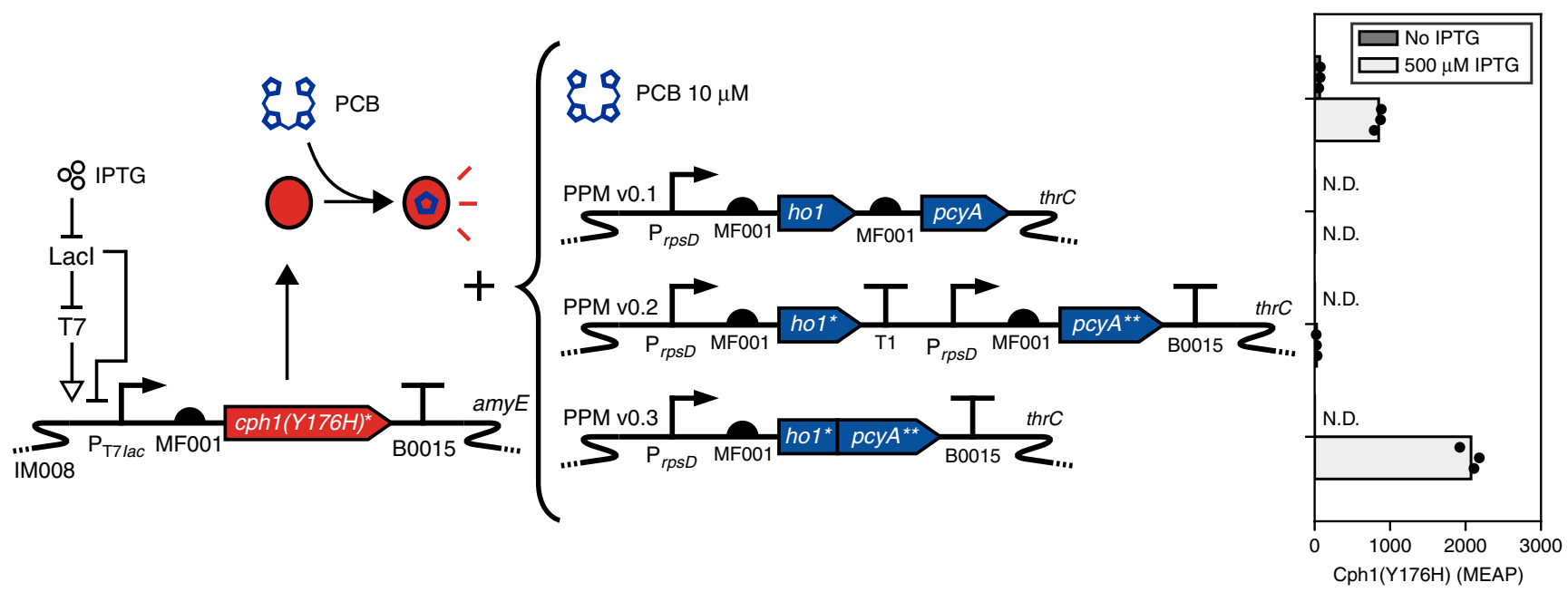

Fig. 2 Debugging and optimizing the PCB production module. B. subtilis expressing $c p h 1(Y 176 H)^{\star}$ from Lacl-T740 were grown in the absence or presence of IPTG, and in the presence of $10 \mu \mathrm{M}$ exogenous PCB, or in combination with PPM v0.1, v0.2, or v0.3. The resulting red fluorescence of the Cph1(Y176H)$\mathrm{PCB}$ conjugate was measured via flow cytometry. Bars show the mean of three experiments run on separate days. Black dots show values of individual experimental replicates. N.D.: not detected (see "Methods" section). Source data is available in the Source Data file

a

$x y / \mathrm{O}_{\mathrm{L}}$

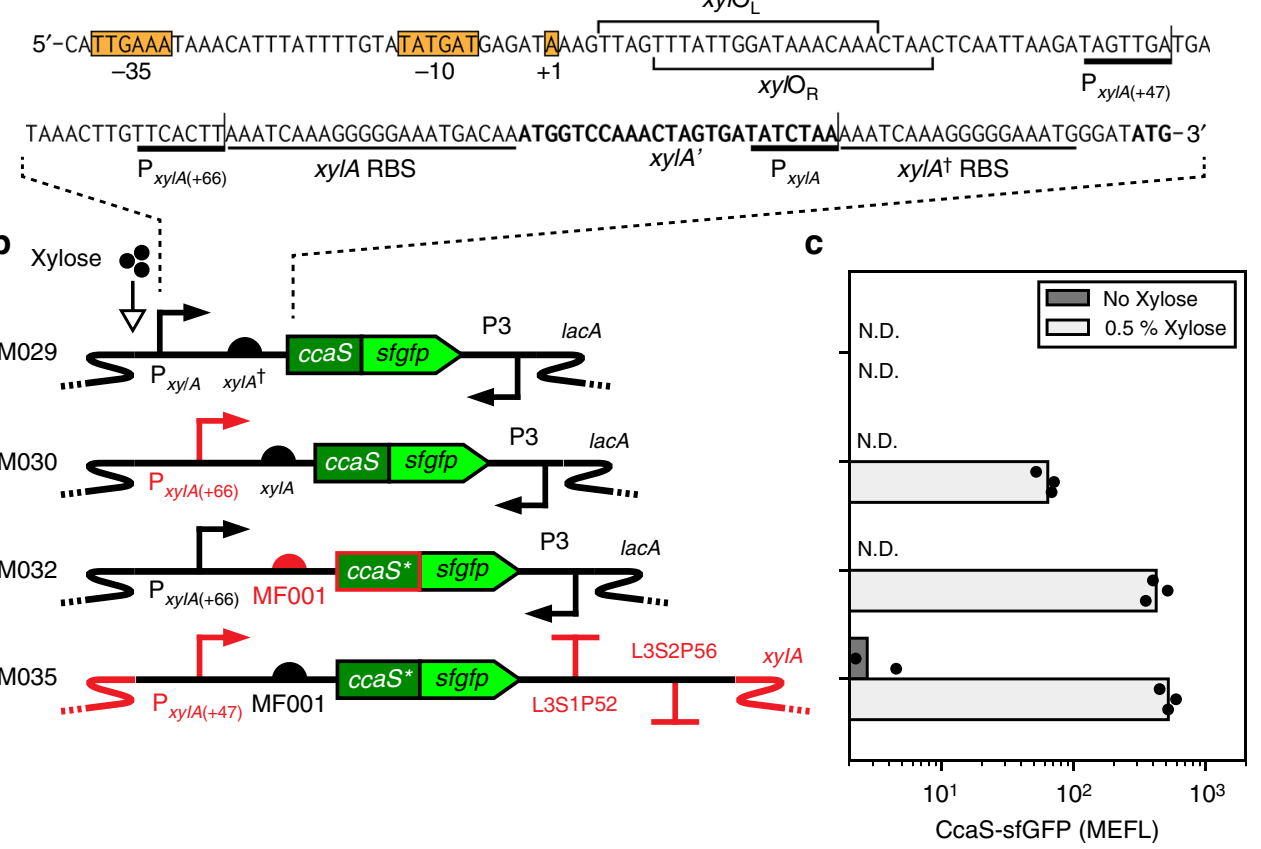

Fig. 3 Debugging and optimization of the light-sensing module. a Annotated sequence of the legacy xylose-inducible promoter $P_{x y l A}$ in LSM v0.1, obtained from integration plasmid $p A X 01^{18,81}$. The $-35,-10$, and +1 sites $^{82}$ and $x y / R$ operators $x y \mid O_{L}$ and $x y \mid O_{R}{ }^{83}$ have been previously identified. The operators are followed by an unwanted untranslated region including the $x y / A$ RBS, and a vestigial truncated $x y / A$ ORF $\left(x y / A^{\prime}\right)$. A second, truncated $x y / A$ RBS ( $\left.x y / A^{\dagger}\right)$ is present in pAX01 to enable translation of a gene of interest placed downstream. Brackets indicate the end of the legacy $P_{x y \mid A}$ promoter, as well as

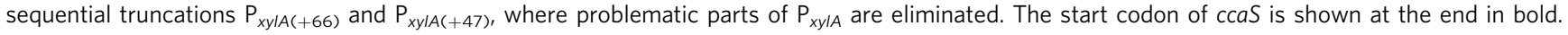
b Measurement and optimization of ccaS expression. We first inserted ccaS-sfgfp as in (a). Next, we truncated $P_{x y l A}$ to remove the vestigial elements (resulting in $\mathrm{P}_{x y / A(+66)}$ ) and placed ccaS-sfgfp directly after the xylA RBS. Then, we switched the xylA RBS with synthetic RBS MF001 (see "Methods" section) and recoded the initial 15 codons of ccaS. Finally, we replaced the vestigial antisense promoter P3 with synthetic terminators L3S1P52 and L3S2P5684, shortened the $P_{x y \mid A(+66)} 5^{\prime}$ UTR (resulting in $P_{x y l A(+47)}$ ), and moved the entire cassette to the xylA chromosomal locus. c Expression of CcaSsfGFP from each engineered module shown in (b). Bars show the mean of three experiments run on separate days. Dots show values of individual experimental replicates. N.D.: not detected (see "Methods" section). Source data is available in the Source Data file

which was more than double the fluorescence that occurred with exogenous PCB addition. Interestingly, levels of Hol-PcyA-sfGFP from PPM v0.3 (19,900 \pm 1100 MEFL, Supplementary Fig. 8) were comparable to those of the non-fused enzymes in PPM v0.2. Thus, the dramatically enhanced PCB levels were not due to higher enzyme expression, but likely to more efficient transfer of BV from Hol to PcyA. We hypothesized that PPM v0.3 would support robust CcaSR function in B. subtilis. 
Debugging and optimizing CcaS expression. We next utilized an $s f g f p$ fusion to analyze ccaS expression from LSM v0.1. We observed no detectable fluorescence (Fig. 3), suggesting that $c c a S$ is also very poorly expressed. A detailed analysis of the corresponding DNA sequence revealed several potentially disruptive elements downstream of $\mathrm{P}_{x y l A}$ that were inherited from our source material, the widely used plasmid pAX01 ${ }^{18}$. In particular, the +1 site and $x y l R$ operators were followed by a 59-bp untranslated region (UTR) containing the xylA RBS, the first five codons of the xylA ORF, a stop codon that prematurely terminates this ORF, a second truncated copy of the xylA RBS, and an antisense promoter (Fig. 3). To eliminate any expression problems that could arise from these elements, we removed the vestigial ORF, the truncated $x y l A$ RBS, and the UTR after the +47 position, swapped in RBS MF001, recoded the initial ccaS ORF as before, and replaced the antisense promoter with terminators. Additionally, to eliminate the possibility of unwanted xylose consumption, we replaced the genomic xylose utilization operon with this new construct (LSM v0.2; Supplementary Fig. 9). As hoped, these changes resulted in a relatively large increase in CcaS expression (520 \pm 74 MEFL) (Fig. 3, Supplementary Fig. 10). We hypothesized that LSM v0.2 would be sufficient for B. subtilis CcaSR function.

Optimizing output promoter activity. Strong maximal transcription is desirable for any engineered promoter system, including our optogenetic system. We next examined the activity of $\mathrm{P}_{c p c G 2-172}$ by comparing it to five $B$. subtilis constitutive promoters of different strengths under control of the house-keeping $\sigma^{\mathrm{A}}$-RNA polymerase ${ }^{36,46,47}$ (see "Methods" section). To measure promoter strength in a standardized manner, we designed a promoter characterization module wherein a self-cleaving ribozyme is followed by RBS MF001 and a sfgfp gene with an initial codon-optimized sequence $\left(s f f f p^{*}\right)$. This design eliminates any differences in mRNA UTR sequences that may arise from the use of different promoters ${ }^{48}$, thus ensuring that sfGFP fluorescence is a function of promoter strength alone (Supplementary Figs. 11, 12). While expression from the five reference promoters ranged from $1076 \pm 100$ MEFL to $133,000 \pm 12,000$ MEFL (Supplementary Fig. 11), maximal $\mathrm{CcaR}$ induction resulted in only $555 \pm 65$ MEFL sfGFP from $P_{c p c G 2-172}$ (Fig. 4, Supplementary Fig. 12). Thus, $\mathrm{P}_{c p c G 2-172}$ is relatively weak in $B$. subtilis.

To increase $\mathrm{P}_{c p c G 2-172}$ activity, we first swapped the original -10 hexamer with the consensus $B$. subtilis sequence to enhance $\sigma^{\mathrm{A}}$-RNA polymerase recruitment. Indeed, this modification increased output expression in the presence of fully induced CcaR nearly 3-fold (1470 \pm 110 MEFL; Fig. 4). To further enhance activity, we then swapped the proximal region, or sequence following the -10 hexamer ${ }^{49}$, with those from the two strongest promoters in our constitutive library, $\mathrm{P}_{r p s D}$ and $\mathrm{P}_{\text {veg. }}$. Indeed, when $c c a R$ is induced, both resulting chimeras $\left(\mathrm{P}_{c p c G 2-r p s D}\right.$ and $\left.\mathrm{P}_{c p c \text { G2-veg }}\right)$ exhibited very strong output $(32,400 \pm 2800 \mathrm{MEFL}$ and $64,000 \pm 7600 \mathrm{MEFL}$, respectively). As expected, we observed low reporter levels when $c c a R$ is not induced $(174 \pm 23$ MEFL and $378 \pm 38$ MEFL, respectively) (Fig. 4, Supplementary Fig. 13). Fold activation values of both promoters in response to IPTG $(186.8 \pm 9.3$ and $169.1 \pm 3.6)$ are not significantly different $(p=$ 0.10 , see "Methods" section). Because output gene expression from a strong promoter can always be brought down via many RBS tuning techniques ${ }^{50,51}$, we chose to replace $\mathrm{P}_{c p c G 2-172}$ in TOM v0.1 with $\mathrm{P}_{c p c G 2-v e g}(\mathrm{TOM}$ v0.2) due to its superior activity.

B. subtilis CcaSR v0.2 responds to light. Next, we integrated PPM v0.3, LSM v0.2, and TOM v0.2 into the chromosome of a single B. subtilis strain, resulting in B. subtilis CcaSR v0.2. As before, we measured green fluorescence from cultures grown at

a

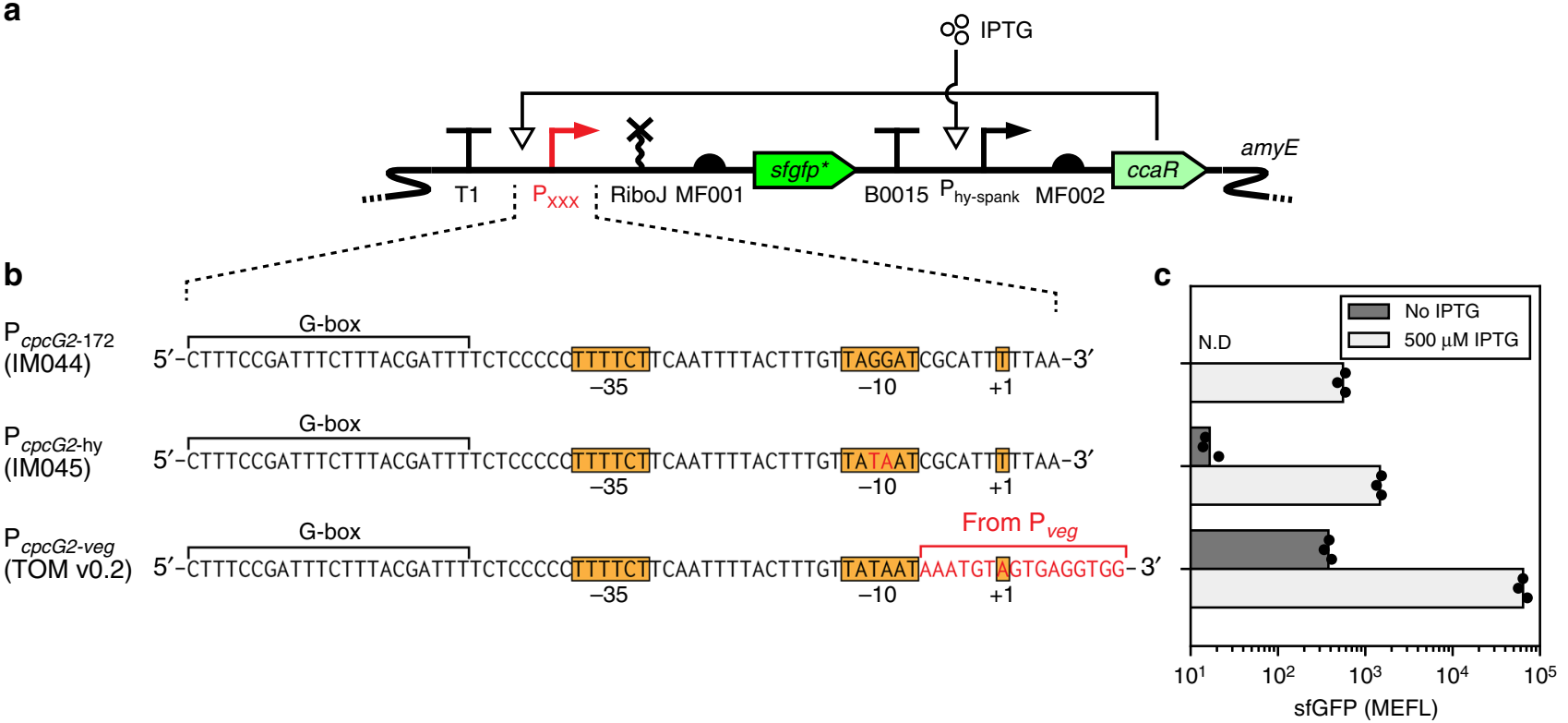

Fig. 4 Optimization of the transcriptional output module. a Characterization of $P_{c p c G 2-172}$ and subsequently-engineered variants using a standard promoter characterization cassette. The promoters are inserted downstream of a transcriptional terminator to prevent any read-through transcription from the amy $E$ locus, and drive transcription of an mRNA containing the self-cleaving ribozyme RiboJ ${ }^{48}$, MF001, and sfgfp with the first 15 codons optimized (sfgfp ${ }^{\star}$ ) (see "Methods" section). IPTG induces ccaR expression, and low-levels of CcaR phosphorylation, likely from a small-molecule phosphoryl donor such as acetyl phosphate ${ }^{37}$, should activate transcription from $P_{c p c G 2-172}$ and further variants. b Regions of interest of $P_{c p c G 2-172}, P_{c p c G 2-h y}$ (named after $P_{s p a c-h y}$, a mutated

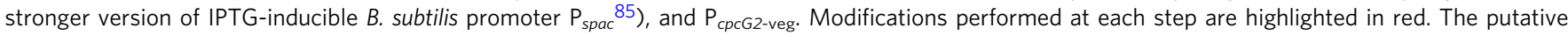
binding region for $\mathrm{CcaR}$ (G-box) is indicated ${ }^{26}$. c sfGFP fluorescence resulting from each output promoter system shown in (b) in the absence and presence of saturating IPTG. Bars show the mean of three experiments run on separate days. Dots show values of individual experimental replicates. N.D.: not detected (see "Methods" section). Source data is available in the Source Data file 


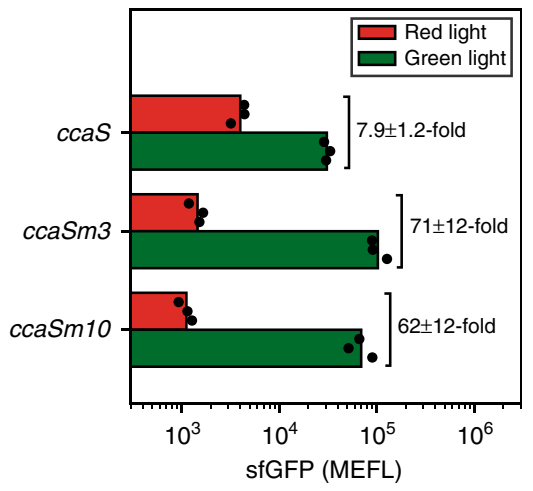

Fig. 5 CcaS miniaturization increases light response. B. subtilis expressing PPM v0.3, TOM v0.2, and either LSM v0.2 (ccaS), LSM v0.3a (ccaSm3), or LSM v0.3b (ccaSm10) were grown at 48 combined levels of IPTG and xylose, under saturating red or green light. sfGFP fluorescence values shown here correspond to the inducer concentrations that resulted in the highest fold-change in response to light for each ccaS variant

(Supplementary Figs. 16, 19, 20). Bars show the mean of three experiments run on separate days. Dots show values of individual experimental replicates. Source data is available in the Source Data file

48 combined xylose and IPTG levels under both red and green light (Supplementary Figs. 14, 16). First, we observed that sfGFP fluorescence increased with $c c a R$ induction, as expected. Additionally, in both red and green light, ccaS induction resulted in an initial increase followed by a large decrease in sfGFP output. We previously observed similar profiles in E. coli, likely due to limiting PCB at high CcaS levels, resulting in Apo-CcaS proteins with phosphatase activity ${ }^{28}$. For a wide range of $c c a S$ and $c c a R$ induction levels, sfGFP levels were higher under green than red light, with a maximum fold-change of $7.9 \pm 1.2$ at $0.0106 \%$ xylose (68.7 MEFL CcaS-sfGFP) and 10.0 $\mu$ M IPTG (537 MEFL CcaRsfGFP) (Supplementary Figs. 14, 16). A control strain lacking PPM v0.3 did not respond to light (Supplementary Figs. 15, 17). Based on these results, we conclude that B. subtilis CcaSR v0.2 functions properly, albeit with low dynamic range.

CcaS miniaturization increases dynamic range. To increase the dynamic range of $B$. subtilis CcaSR v0.2, we separately replaced ccaS in LSM v0.2 with mini-ccaS\#3 and mini-ccaS\#10 (hereafter ccaSm3 and ccaSm10), two miniaturized variants that exhibit enhanced dynamic range in $E$. coli ${ }^{29,30}$. Indeed, both of the variants increased dynamic range approximately 10-fold (ccaSm3: $71 \pm 12$-fold, ccaSm10: $62 \pm 12$-fold) (Fig. 5, Supplementary Figs. 19, 20). In contrast to E. coli, where ccaSm 10 resulted in a much larger dynamic range, both variants give rise to statistically identical values in B. subtilis ( $p=0.49$, see "Methods" section). In both organisms, however, ccaSm10 results in overall lower output expression levels compared to $c c a S m 3$. The reason why this only results in a larger fold-change in E. coli is currently unknown. Due to its superior performance, we named the system containing ccaSm3 B. subtilis CcaSR v1.0, and carried it forward for further characterization.

Characterization of B. subtilis CcaSR v1.0 Light Response. To compare B. subtilis CcaSR v1.0 activity to that of our E. coli systems, we measured the steady-state transfer function (Fig. 6b) by growing the engineered strain under varying intensities of green light (see "Methods" section). With increasing green light intensity, sfGFP gradually increased from $2000 \pm 190$ to $108,700 \pm 9200$ MEFL in a manner well approximated by a Hill function. Fits to the experimental data revealed a Hill coefficient of $1.88 \pm 0.16$ and a $50 \%$ activation intensity of $4.66 \pm 0.63 \mu \mathrm{mol}$ $\mathrm{m}^{-2} \mathrm{~s}^{-1}$ (Fig. 6b). Thus, B. subtilis CcaSR v1.0 exhibits analog green light-intensity dependent transcriptional output, as our $E$. coli systems do. Interestingly, this transfer function was more gradual and the $50 \%$ activation intensity was more than four times higher than in the E. coli system (Hill coefficient: $2.737 \pm$ $0.044,50 \%$ activation intensity: $\left.1.075 \pm 0.025 \mu \mathrm{mol} \mathrm{m}^{-2} \mathrm{~s}^{-1}\right)^{30}$. The origins of these differences are not immediately clear and warrant further investigation.

Finally, we characterized the response dynamics of CcaSR after an instantaneous switch from saturating red light to green light (Fig. 6c) or vice-versa (Fig. 6d). Both responses showed a short delay followed by an exponential-like increase or decrease in sfGFP fluorescence until saturation, with half-maximum activation $\left(\mathrm{t}_{1 / 2}{ }^{\text {on }}\right)$ and deactivation $\left(\mathrm{t}_{1 / 2}{ }^{\text {off }}\right)$ times of $105.1 \pm 1.5$ and $74.97 \pm 0.39 \mathrm{~min}$, respectively (see "Methods" section). These response times are similar to those observed in $E$. coli, albeit slightly slower $\left(t_{1 / 2} \text { on }=77 \mathrm{~min}, t_{1 / 2} \text { off }=62 \mathrm{~min}\right)^{30}$. To explain this difference, we note that in our previous $E$. coli work we have used an antibiotic protocol to arrest cell growth and protein synthesis and allow maturation of all intracellular sfGFP ${ }^{20}$. Because all sfGFP was mature at the time of measurement, the effect of sfGFP maturation kinetics in the observed dynamics was eliminated. In contrast, we have been unable to develop a similar B. subtilis maturation protocol. Therefore, sfGFP maturation kinetics slow the observed response dynamics ${ }^{52}$, especially as expression increases and more immature sfGFP is produced. Thus, these results show that CcaSR exhibits similar dynamic I/O properties in B. subtilis and E. coli.

\section{Discussion}

We have engineered CcaSR as the first optogenetic tool for $B$. subtilis, the leading model Gram-positive bacterium. CBCRs, and their bilin-binding relatives the phytochromes, tend to respond to lower intensities of light than other commonly used optogenetic tools, such as the flavin-binding LOV domain or Cryptochrome $2^{53}$. Although slightly greater light intensities are required to activate CcaSR in B. subtilis as compared to E. coli, they are still within the range of intensities used to modulate most other bacterial optogenetic tools ${ }^{54-57}$. Furthermore, these do not lead to phototoxic effects in B. subtilis (Supplementary Fig. 21).

One of the challenges when porting a bilin-binding photoreceptor into a heterologous host organism is chromophore availability. PcyA has evolved to utilize cyanobacterial or plant ferredoxins to produce PCB, and it has been shown to work poorly with Gram-positive ferredoxins ${ }^{58}$. Recently, two groups achieved efficient PCB production in mammalian cells by expressing a plant ferredoxin and a ferredoxin-NADPH reductase in addition to hol and $p c y A^{59,60}$. In contrast, here we showed that a translational ho1-pcyA fusion can dramatically increase PCB production without additional enzymes. Theoretical models suggest that colocalization alone should not improve yield unless multiple copies of each enzyme are clustered ${ }^{35,61}$, or the enzymes naturally engage in substrate channeling, the transfer of intermediate metabolites via protein-protein interaction ${ }^{62}$. Interestingly, channeling may naturally occur between human heme oxygenase and biliverdin reductase in the human heme catabolic pathway ${ }^{63}$. Our results are consistent with Hol and PcyA engaging in substrate channeling, where the flexible linker increases the rate of BV transfer and therefore the metabolic flux. Translational fusions may be a general method to increase biosynthetic pathway yields in cellular environments where some accessory proteins are lacking.

As in E. coli, CcaSR should enable unrivaled temporal and spatial control of gene expression in B. subtilis. In particular, by 

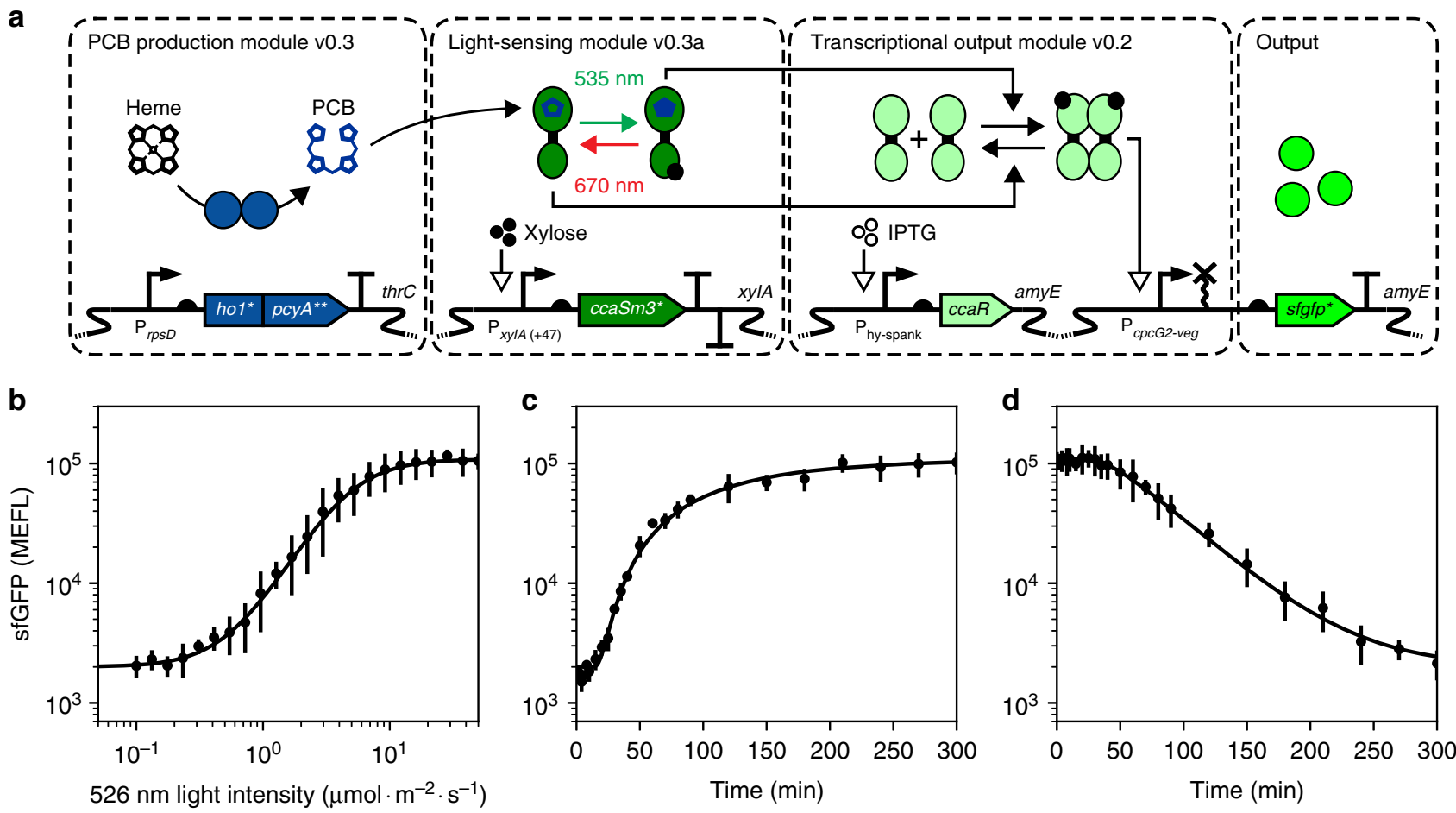

Fig. 6 Characterization of B. subtilis CcaSR v1.0 input/output properties. a Schematic of the final B. subtilis CcaSR v1.0 design. b Steady-state transfer function. c Step ON response. d Step OFF response. Dots and error bars show the mean and standard deviation, respectively, of three experiments run on separate days. Black lines represent model fits (see "Methods" section). Source data is available in the Source Data file

conducting additional step ON and step OFF kinetic measurements at different initial and final light intensities, we should be able to construct a general mathematical model of how CcaSR output gene expression changes over time in response to any time-varying light input signal, following our established methods $^{20}$. Then, we can utilize our biological function generator method to design light signals capable of driving sophisticated gene expression signals with quantitative accuracy ${ }^{20}$.

By encoding regulatory proteins as the output of CcaSR, such signals can be used as a tool to interrogate the dynamical signal processing properties of important cellular decision-making networks such as those involved in stress response, sporulation, and biofilm formation. For example, while the mechanism by which pulses of the master sporulation regulator Spo0A arise has been elucidated 8,9 , their impact on downstream processes remains unclear. Interestingly, the use of a chemically-inducible promoter to express the constitutively-active mutant Spo0Asad67 does not trigger sporulation ${ }^{64}$. It has been proposed that fast Spo0A activation leads to early repression of genes that are essential for sporulation, thus leading to non-viable spore formation $^{65}$. CcaSR could be used to create different dynamical expression patterns of Spo0A-sad67, such as ramps of different slopes and pulses of different amplitudes, frequencies, or phases, in order to understand how the downstream sporulation circuit responds to Spo0A activity dynamics. Moreover, the ability to use light to control gene expression in individual cells could be exploited when studying processes involving noisy or spatiallydelineated gene expression patterns.

We have recently characterized the full spectral response properties of CcaSR and the related red/far-red photoreversible sensor Cph8-OmpR to overcome inherent spectral overlap between the systems and achieve simultaneous and independent control of the expression dynamics of two separate genes in E. coli $^{32}$. We believe Cph8-OmpR or other bacterial optogenetic tools $54,55,57,66$ could also be ported into B. subtilis and combined with CcaSR to achieved multiplexed control of gene expression dynamics. Such a technology would be particularly useful in examining whether $B$. subtilis gene circuits require specific combinations of dynamical gene expression signals to function properly $^{22}$.

This work also advances $B$. subtilis synthetic biology in several ways. First, our results suggest that genes ported into B. subtilis from other organisms may frequently be inefficiently translated due to mRNA secondary structure between the RBS and initial ORF sequence. Since strong B. subtilis RBSs seem to have little sequence variation ${ }^{43}$, RBS redesign to reduce secondary structure might not always be feasible (Supplementary Fig. 22). On the other hand, several studies have shown that strongly expressed bacterial genes have an initial codon bias that minimizes secondary structure ${ }^{41-43}$. Furthermore, secondary structure entirely contained within the initial ORF sequence has been found to greatly affect translation as well ${ }^{44}$. All cyanobacterial genes in this study, apart from $c c a R$, suffered initially from poor expression. In all cases, we showed that codon optimization of the initial 15 amino acids can result in dramatic expression improvements (Supplementary Fig. 23). A computational tool that modifies an ORF sequence to tune translation currently exists only for $E$. coli $^{67}$. Our results indicate that a tool for B. subtilis that operates under the same principles is feasible. However, validation against a large variety of genes and expression cassettes would be necessary.

We also show that proper insulation from the genomic context, in the form of a transcriptional terminator at the 3' of an expression cassette, leads to a dramatic increase in gene expression in at least two of the most widely-used genomic integration loci: the thrC locus (Supplementary Fig. 5, 21-fold PcyA expression difference) and the amyE locus (Supplementary Fig. 7, 11fold sfGFP expression difference). While at least one study 
recognizes the need for a well-insulated expression cassette in $B$. subtilis $^{46}$, plasmids for C-terminal fusions to lacZ or other reporter genes offered by the Bacillus Genetic Stock Center are not properly insulated. It is possible that incorporation of a single terminator could greatly improve the limit of detection of future assays based on these plasmids.

The sequence determinants of the strength of promoters controlled by the housekeeping sigma factor $\sigma^{A}$ are classically thought to be the -35 and -10 hexamers, and to a lesser extent a -16 region and a $\mathrm{A} / \mathrm{T}$ rich sequence upstream of the -35 hexamer ${ }^{68-70}$. Here we show that the sequence downstream of the -10 hexamer can greatly affect transcription as well. We found that $\mathrm{P}_{c p c G 2-172}$ output was low compared to a small library of constitutive promoters. To engineer a stronger promoter while keeping the CcaR operators intact, we swapped the sequence after the -10 hexamer with sequences from strong constitutive promoters $\mathrm{P}_{r p s D}$ and $\mathrm{P}_{\text {veg. }}$. These mutations increased output transcription by 22 - and 44 -fold, respectively. A larger scale study to explore the generality of this technique would be of great interest to $B$. subtilis synthetic biology practitioners, particularly of those that work in engineering transcription factor-inducible promoters, where the transcription factor binding site covers everything but the sequence after the -10 hexamer.

In conclusion, we have engineered the first, to our knowledge, B. subtilis optogenetic system. B. subtilis CcaSR v1.0 should enable precise temporal and spatial control of gene expression in $B$. subtilis, and thus be widely used to study complex cellular processes such as sporulation, stress response, and biofilm formation, among others. We have also unveiled several design principles that should be of great utility in future B. subtilis synthetic biology applications.

\section{Methods}

DNA and strain construction. All cloning and experiments were performed in $B$. subtilis strain PY79. A list of all strains constructed in this study can be found in Supplementary Data 1. Strains with each one of the final CcaSR modules (PPM v0.3, LSM v0.3a, TOM v0.2), the complete CcaSR v1.0 system, and the optimized $\operatorname{cph} 1(\mathrm{Y} 176 \mathrm{H})^{*} \mathrm{PCB}-$ dependent fluorescent protein can be obtained from the Bacillus Genetic Stock Center via the specified accession numbers. All other strains are available from the corresponding author on reasonable request.

Primers were ordered from Integrated DNA Technologies, Inc. All TCS genes were amplified from previous E. coli CcaSR plasmids ${ }^{28}$. Cph1 $(\mathrm{Y} 176 \mathrm{H})$ was amplified from the Cph1-EnvZ chimera Cph8 in pSR33.4 ${ }^{28} . p c y A^{* *}$ was designed using GeneOptimizer ${ }^{71}$ and ordered as a gBlock from Integrated DNA Technologies, Inc. The xylose and IPTG-inducible cassettes were amplified from integration plasmids $\mathrm{pAX} 01^{18}$ and $\mathrm{pDR} 111^{19}$. Constitutive promoters were constructed via oligo annealing and extension. Sequences of $\mathrm{P}_{\text {liaG }}, \mathrm{P}_{\text {lepA }}$, and $\mathrm{P}_{\text {veg }}$ comprise bases from the -66 to the $+10\left(\mathrm{P}_{\text {liaG }}, \mathrm{P}_{\text {veg }}\right)$ or $+12\left(\mathrm{P}_{\text {lepA }}\right)$ position of the corresponding promoters of the BioBricks library ${ }^{47}$. The sequence of $\mathrm{P}_{r p s D}$ comprises bases from the -58 to the +16 position of the corresponding chromosomal promoter ${ }^{36} . \mathrm{P}_{\mathrm{S}^{*}}$ is identical to $\mathrm{P}_{\text {sarA }}$-derived $\mathrm{P}_{\mathrm{S} 1}{ }^{46}$, but with the unmodified $P_{\text {sarA }}$ 5'UTR. Synthetic RBS MF001 was obtained from integration plasmid pMF $35^{72}$. Genomic homology fragments required for chromosomal integration were amplified from the purified genome of B. subtilis PY79. A list of genetic parts, along with their sequences, can be found in Supplementary Data 2.

All systems were built as linear double-stranded integration modules (IMs) ${ }^{73}$. The IMs contain the DNA of interest and a selection marker flanked by $1.5 \mathrm{~kb}-$ long sequences homologous to a region of the B. subtilis genome (integration locus) where chromosomal integration via double crossover occurs. IMs were assembled from PCR-amplified parts using GoldenGate ${ }^{74}$. The resulting Golden Gate products were amplified using NEB Phusion DNA Polymerase and gel purified to obtain the IM. $500 \mathrm{ng}$ or more of each IM was transformed into competent $B$. subtilis using standard transformation methods. The transformants were plated on selective media. Colonies were picked the next day and grown in LB media at $37^{\circ} \mathrm{C}$ and 250 RPM for $\sim 2 \mathrm{~h}$ until turbidity is visible. Finally, freezer stocks were prepared with $700 \mu \mathrm{L}$ culture and $300 \mu \mathrm{L} 60 \%$ glycerol, and stored at $-80{ }^{\circ} \mathrm{C}$. This method avoids subcloning of integration plasmids in E. coli, as long as enough PCR-amplified DNA can be obtained. A list of all IMs constructed in this study can be found in Supplementary Data 3, and their complete sequences can be found on genbank via the specified accession numbers.

For sequence verification, an overnight LB culture was grown from a freezer stock, and $2 \mu \mathrm{L}$ saturated culture was used as template for a $50 \mu \mathrm{L}$ PCR reaction, either with Taq or Phusion DNA Polymerase. PCR products obtained in this fashion were gel-purified and sent for sequence verification to Genewiz, Inc.

To construct strains with two IMs, two separate strains containing each IM were cloned and sequence-verified independently, as described above. Next, one of these strains was made competent, and the genomic DNA of the second was extracted from an overnight culture using the Promega Wizard Genomic DNA Purification Kit. Next, 500-1000 ng of purified genomic DNA was transformed into the competent strain as described above to produce a new strain with both IMs. Correct integration of both modules was verified via PCR. If necessary, a strain with two IMs was made competent and a third IM was integrated via the same procedure.

Codon optimization. For each of the first 15 codons, a synonymous codon was chosen to reduce GC and increase AU content, with A preferred over U, with no regard for codon frequency. The free energy of the resulting mRNA secondary structure was calculated via Nupack ${ }^{75}$ using the sequence from the transcription start site up to the 90th nucleotide residue of the ORF.

Media and experimental protocols. We used a modified M9 media for experiments. $1 \mathrm{~L} 5 \times \mathrm{M} 9$ salts at $\mathrm{pH} \sim 6.8$ were prepared with $64 \mathrm{~g} \mathrm{Na}_{2} \mathrm{HPO}_{4} .7 \mathrm{H}_{2} \mathrm{O}, 15 \mathrm{~g}$

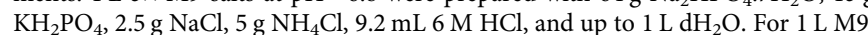
we used $200 \mathrm{~mL} 5 \times$ M9 salts, $20 \mathrm{~mL} \mathrm{10 \%} \mathrm{casamino} \mathrm{acids,} 6.67 \mathrm{~mL} 60 \%$ glycerol, $1 \mathrm{~mL} 50 \mathrm{mM} \mathrm{FeCl} / 100 \mathrm{mM} \mathrm{C}_{6} \mathrm{H}_{8} \mathrm{O}_{7}$ solution, $2 \mathrm{~mL} 50 \mathrm{mM} \mathrm{MnSO}$, $2 \mathrm{~mL} 1 \mathrm{M}$ $\mathrm{MgSO}_{4}, 100 \mu \mathrm{L} 1 \mathrm{M} \mathrm{CaCl}_{2}$, and $\mathrm{dH}_{2} \mathrm{O}$ up to $1 \mathrm{~L}$. Glycerol was used as a carbon source since glucose strongly represses expression from $\mathrm{P}_{x y l A}$. The $\mathrm{FeCl}_{3}$ solution appears necessary to support robust growth of PCB-producing strains.

For every experiment, an overnight LB culture was started from the freezer stock of each relevant strain. The next day, saturated cultures (OD600 3) were diluted $10^{4}-10^{5}$-fold in M9. For non-optogenetic experiments, media was distributed in culture tubes ( $3 \mathrm{~mL}$ per tube), inoculated with the appropriate inducers, and incubated in a shaker operating at $250 \mathrm{RPM}$ and $37^{\circ} \mathrm{C}$ until the $\mathrm{OD}_{600}$ reached between 0.08 and 0.15 (6-9 h depending on initial density and strain). When indicated, saturating concentrations of xylose (0.5\%) and IPTG $(500 \mu \mathrm{M})$ were used. Inducer concentrations used in Fig. 6 were $0.00917 \%$ xylose and $10.0 \mu \mathrm{M}$ IPTG. In optogenetic experiments, media was distributed $(500 \mu \mathrm{L}$ per well) in 24-well dark-walled clear-bottomed plates (ArcticWhite AWLS303008). Next, the appropriate inducers were added, and plates were sealed with adhesive foil (VWR 60941-126). Plates were mounted onto LPAs running the appropriate light exposure programs, and incubated until the $\mathrm{OD}_{600}$ reached between 0.08 and 0.15 . Culture tubes or plates were then transferred to ice. $100 \mu \mathrm{L}$ of each sample was transferred to a flow cytometry tube containing $1 \mathrm{~mL}$ PBS for measurement.

PCB was purified from Spirulina powder using methanolysis, as previously described $^{76}$. Samples, where purified PCB was added, were incubated in culture tubes at $250 \mathrm{RPM}$ and $37^{\circ} \mathrm{C}$ for $5 \mathrm{~h}$. Next, $10 \mu \mathrm{M}$ purified PCB was added under a green safelight, and tubes were returned to the incubator for $1 \mathrm{~h}$. At the end of the experiment, tubes were placed on ice and $\mathrm{Cph} 1(\mathrm{Y} 176 \mathrm{H})$ fluorescence was measured via flow cytometry.

Optical hardware. Eight 24-well Light-Plate Apparatuses (LPAs) ${ }^{77}$ equipped with green (520-2-KB, WP7083ZGD/G, Kingbright, CA, USA) and red (660-LS, L2-0R5TH50-1, LEDSupply, VT, USA) LEDs were used for all optogenetic experiments. These were mounted in a shaking incubator operating at $250 \mathrm{RPM}$ and $37^{\circ} \mathrm{C}$. Total LED power output in $\mu \mathrm{mol} \mathrm{s}^{-1}$ was measured using a spectrometer (StellarNet UVN-SR-25 LT16) attached to a six-inch integrating sphere (StellarNet IS6). The average light intensity was calculated by dividing the total power output by the area of a circular plate well with a radius of $7.5 \mathrm{~mm}$, as previously ${ }^{32}$. To calibrate the power output of each LED, we adjusted the current using the LPA Dot Correction setting to achieve saturating intensities of $20 \mu \mathrm{mol} \mathrm{m}^{-2} \mathrm{~s}^{-1}$ or more for red LEDs and $50 \mu \mathrm{mol} \mathrm{m}^{-2} \mathrm{~s}^{-1}$ or more for green LEDs. Each LED was measured while powered from the same LPA socket used in experiments. The precisely measured intensities and dot correction values were recorded. Custom Python scripts were written to use these recordings to achieve light intensities necessary in each experiment.

Flow cytometry analysis. The sfGFP fluorescence distribution of each B. subtilis culture sample was measured using a BD FACScan flow cytometer with an excitation source of $488 \mathrm{~nm}$ and an emission window of 510/21 nm. 10,000-30,000 events were collected per sample. A suspension of calibration beads (Spherotech RCP-30-5A) in PBS was measured with each experiment. After data acquisition, raw.fcs flow cytometry files were processed using $\mathrm{FlowCal}^{78}$. Cell populations were gated by forward scatter/side scatter density (Supplementary Fig. 24), retaining $50 \%$ of the total number of events. Next, fluorescence of each gated event in arbitrary units was converted into standardized MEFL (Molecules of Equivalent Fluorescein) values using the calibration bead data. The cell fluorescence of each culture sample was then obtained from the median MEFL fluorescence of all gated events in that sample. Finally, the sfGFP fluorescence was obtained by subtracting the cell fluorescence of each sample from the cell fluorescence of a control sample lacking any fluorescent reporter gene measured the same day (autofluorescence control). The strain used as an autofluorescence control for any strain containing 
PPM v0.3 was a strain containing the PPM v0.3 only. The autofluorescence strain for any other strain was the wild-type PY79.

The single cell $\mathrm{Cph} 1(\mathrm{Y} 176 \mathrm{H})$ fluorescence distribution was measured using a BD FACSCanto II. The fluorescent channel used (APC-A) used a $633 \mathrm{~nm}$ red laser as the excitation source, and an emission window of $670 / 50 \mathrm{~nm}$. Calibration beads were measured as above. FlowCal was used to gate cell populations and calibrate fluorescence data to MEAP (Molecules of Equivalent Allophycocyanin). The cell fluorescence of each culture sample was then obtained from the median MEAP fluorescence of all gated events in that sample. Finally, the Cph1 $(\mathrm{Y} 176 \mathrm{H})$ fluorescence was obtained by subtracting the cell fluorescence of each sample from the cell fluorescence of a control wild-type PY79 sample.

Statistical analysis. Each experiment was replicated three times over different days. Fluorescence of each sample is reported as the mean \pm std dev. of the sfGFP and $\mathrm{Cph} 1(\mathrm{Y} 176 \mathrm{H})$ fluorescence from three experiments. A one-sample Student's $t$ test was conducted for every sample to evaluate whether sfGFP or Cph1 $(\mathrm{Y} 176 \mathrm{H})$ fluorescence was significantly different from zero (one-sided, $p<0.05$ ). Fluorescence of samples that failed this test are reported as not detected or N.D. Significance in the difference of fold changes was calculated via a two-sample Welch's unequal variances $t$-test.

Transfer function modeling and fitting. Steady-state transfer functions in Fig. 6b, Supplementary Figs. 14, 15, and 18 were fitted to a Hill Function of the form:

$$
y=y_{0}+\Delta y \cdot \frac{x^{n}}{x^{n}+K_{1 / 2}^{n}}
$$

Here, $y$ is the observed sfGFP fluorescence in MEFL, which has a minimum value of $y_{0}$ in the absence of inducer and a maximum of $y_{0}+\Delta y$ under saturating conditions, $x$ is either light intensity in $\mu \mathrm{mol} \mathrm{m}^{-2} \mathrm{~s}^{-1}$ (Fig. 6b), Xylose concentration in \% (Supplementary Figs. 14B, 15B, and 18) or IPTG concentration in $\mu \mathrm{M}$ (Supplementary Figs. $14 \mathrm{D}$ and $15 \mathrm{D}$ ), $K_{1 / 2}$ is the inducer concentration for half-maximum activation, and $n$ is the Hill coefficient. Fitting was performed using the LmFit python package ${ }^{79}$ with the Levenberg-Marquardt algorithm.

Experimental data from three replicates were combined and fitted simultaneously. To adequately fit low and high fluorescence values, the error to minimize was defined as the difference between the logarithm of a fluorescence datapoint and the logarithm of the model prediction. Fitted parameter values and their uncertainties can be found in Supplementary Data 4.

Kinetic response modeling and fitting. The response of the CaSR system to an instantaneous change in light intensity was modeled as a differential equation system of the form:

$$
\begin{gathered}
\frac{d p}{d t}=k_{p} \cdot\left(c\left(I_{g}, I_{r}\right)-p(t)\right) \\
\frac{d g}{d t}=k_{g} \cdot(p(t-\tau)-g(t)) \\
\frac{d G}{d t}=k_{d} \cdot(g(t)-G(t))
\end{gathered}
$$

Here, $p(t)$ represents the sfGFP production rate, $g(t)$ represents immature sfGFP, and $G(t)$ is the fully mature, observed sfGFP. Their dynamics are determined by rate constants $k_{p}, k_{g}$, and $k_{G}$. $\tau$ represents the delay before a change in sfGFP production actually occurs. Finally, $c$ is the system input, and is a function of light intensities $I_{g}$ and $I_{r}$. Units for $c, p(t)$, and $G(t)$ have been chosen such that, in steady state, $c=p_{s s}=$ $g_{s s}=G_{s s}$ and thus $c$ determines the steady state output fluorescence. To simulate an instantaneous change from red to green light (Fig. $6 \mathrm{c}$ ), the model was solved with initial conditions $p(0)=g(0)=G(0)=y_{0}$ and $c=y_{0}+\Delta y$, where $y_{0}$ and $\Delta y$ are obtained from the Hill function fit. Similarly, an instantaneous change from green to red light (Fig. 6d) was simulated with $p(0)=g(0)=G(0)=y_{0}+\Delta y$ and $c=y_{0}$. Fitting was performed using LmFit as described above.

Reporting summary. Further information on research design is available in the Nature Research Reporting Summary linked to this article.

\section{Data availability}

Sequences of all integration modules used in this study are available from Genbank via the accession numbers listed in Supplementary Data 3. Flow cytometry data and scripts used to generate all figures are available from figshare (https://doi.org/10.6084/m9. figshare.8198999) ${ }^{80}$. Parameters of Hill function fits can be found in Supplementary Data 4. Source data for Figs. 2-6 and Supplementary Figs. 1-8, 10-22 can be found in the Source Data file. Any other relevant data can be obtained from the authors upon reasonable request.

Received: 9 November 2018 Accepted: 7 June 2019

Published online: 15 July 2019

\section{References}

1. Levine, J. H., Lin, Y. \& Elowitz, M. B. Functional roles of pulsing in genetic circuits. Science 342, 1193-1200 (2013).

2. Vlamakis, H., Chai, Y., Beauregard, P., Losick, R. \& Kolter, R. Sticking together: building a biofilm the Bacillus subtilis way. Nat. Rev. Microbiol. 11 157-168 (2013).

3. González-Pastor, J. E. Cannibalism: a social behavior in sporulating Bacillus subtilis. FEMS Microbiol. Rev. 35, 415-424 (2011).

4. Narula, J., Fujita, M. \& Igoshin, O. A. Functional requirements of cellular differentiation: lessons from Bacillus subtilis. Curr. Opin. Microbiol. 34, 38-46 (2016).

5. Locke, J. C. W., Young, J. W., Fontes, M., Jiménez, M. J. H. \& Elowitz, M. B. Stochastic pulse regulation in bacterial stress response. Science 334, 366-369 (2011).

6. Young, J. W., Locke, J. C. W. \& Elowitz, M. B. Rate of environmental change determines stress response specificity. Proc. Natl Acad. Sci. 110, 4140-4145 (2013).

7. Levine, J. H., Fontes, M. E., Dworkin, J. \& Elowitz, M. B. Pulsed feedback defers cellular differentiation. PLOS Biol. 10, e1001252 (2012).

8. Narula, J. et al. Chromosomal arrangement of phosphorelay genes couples sporulation and dna replication. Cell 162, 328-337 (2015).

9. Narula, J. et al. Slowdown of growth controls cellular differentiation. Mol. Syst. Biol. 12, 871 (2016).

10. Veening, J.-W. et al. Bet-hedging and epigenetic inheritance in bacterial cell development. Proc. Natl Acad. Sci. USA 105, 4393-4398 (2008).

11. Süel, G. M., Garcia-Ojalvo, J., Liberman, L. M. \& Elowitz, M. B. An excitable gene regulatory circuit induces transient cellular differentiation. Nature 440, 545-550 (2006).

12. Süel, G. M., Kulkarni, R. P., Dworkin, J., Garcia-Ojalvo, J. \& Elowitz, M. B. Tunability and noise dependence in differentiation dynamics. Science $\mathbf{3 1 5}$, 1716-1719 (2007).

13. Kuchina, A. et al. Temporal competition between differentiation programs determines cell fate choice. Mol. Syst. Biol. 7, 557 (2011).

14. Vlamakis, H., Aguilar, C., Losick, R. \& Kolter, R. Control of cell fate by the formation of an architecturally complex bacterial community. Genes Dev. 22, 945-953 (2008).

15. Liu, J. et al. Metabolic co-dependence gives rise to collective oscillations within biofilms. Nature 523, 550-554 (2015).

16. Prindle, A. et al. Ion channels enable electrical communication in bacterial communities. Nature 527, 59-63 (2015).

17. Koo, B.-M. et al. Construction and analysis of two genome-scale deletion libraries for Bacillus subtilis. Cell Syst. 4, 291-305.e7 (2017).

18. Härtl, B., Wehrl, W., Wiegert, T., Homuth, G. \& Schumann, W. Development of a new integration site within the Bacillus subtilis chromosome and construction of compatible expression cassettes. J. Bacteriol. 183, 2696-2699 (2001).

19. Britton, R. A. et al. Genome-wide analysis of the stationary-phase sigma factor (Sigma-H) regulon of Bacillus subtilis. J. Bacteriol. 184, 4881-4890 (2002).

20. Olson, E. J., Hartsough, L. A., Landry, B. P., Shroff, R. \& Tabor, J. J. Characterizing bacterial gene circuit dynamics with optically programmed gene expression signals. Nat. Methods 11, 449-455 (2014).

21. Chait, R., Ruess, J., Bergmiller, T., Tkačik, G. \& Guet, C. C. Shaping bacterial population behavior through computer-interfaced control of individual cells. Nat. Commun. 8, 1535 (2017).

22. Castillo-Hair, S. M., Igoshin, O. A. \& Tabor, J. J. How to train your microbe: methods for dynamically characterizing gene networks. Curr. Opin. Microbiol. 24, 113-123 (2015).

23. Hirose, Y., Shimada, T., Narikawa, R., Katayama, M. \& Ikeuchi, M. Cyanobacteriochrome $\mathrm{CcaS}$ is the green light receptor that induces the expression of phycobilisome linker protein. Proc. Natl Acad. Sci. 105, 9528-9533 (2008)

24. Frankenberg, N., Mukougawa, K., Kohchi, T. \& Lagarias, J. C. Functional genomic analysis of the HY2 family of ferredoxin-dependent bilin reductases from oxygenic photosynthetic organisms. Plant Cell Online 13, 965-978 (2001).

25. Gambetta, G. A. \& Lagarias, J. C. Genetic engineering of phytochrome biosynthesis in bacteria. Proc. Natl Acad. Sci. USA 98, 10566-10571 (2001).

26. Hirose, Y., Narikawa, R., Katayama, M. \& Ikeuchi, M. Cyanobacteriochrome CcaS regulates phycoerythrin accumulation in Nostoc punctiforme, a group II chromatic adapter. Proc. Natl Acad. Sci. 107, 8854-8859 (2010).

27. Tabor, J. J., Levskaya, A. \& Voigt, C. A. Multichromatic control of gene expression in Escherichia coli. J. Mol. Biol. 405, 315-324 (2011).

28. Schmidl, S. R., Sheth, R. U., Wu, A. \& Tabor, J. J. Refactoring and optimization of light-switchable Escherichia coli two-component systems. ACS Synth. Biol. 3, 820-831 (2014).

29. Nakajima, M., Ferri, S., Rögner, M. \& Sode, K. Construction of a miniaturized chromatic acclimation sensor from Cyanobacteria with reversed response to a light signal. Sci. Rep. 6, 37595 (2016). 
30. Ong, N. T. \& Tabor, J. J. A Miniaturized Escherichia coli green light sensor with high dynamic range. ChemBioChem 19, 1255-1258 (2018)

31. Fernandez-Rodriguez, J., Moser, F., Song, M. \& Voigt, C. A. Engineering RGB color vision into Escherichia coli. Nat. Chem. Biol. 13, 706 (2017).

32. Olson, E. J., Tzouanas, C. N. \& Tabor, J. J. A photoconversion model for full spectral programming and multiplexing of optogenetic systems. Mol. Syst. Biol. 13, 926 (2017).

33. Milias-Argeitis, A., Rullan, M., Aoki, S. K., Buchmann, P. \& Khammash, M. Automated optogenetic feedback control for precise and robust regulation of gene expression and cell growth. Nat. Commun. 7, 12546 (2016).

34. Conrado, R. J., Varner, J. D. \& DeLisa, M. P. Engineering the spatial organization of metabolic enzymes: mimicking nature's synergy. Curr. Opin. Biotechnol. 19, 492-499 (2008)

35. Lee, H., DeLoache, W. C. \& Dueber, J. E. Spatial organization of enzymes for metabolic engineering. Metab. Eng. 14, 242-251 (2012).

36. Grundy, F. J. \& Henkin, T. M. Characterization of the Bacillus subtilis rpsD regulatory target site. J. Bacteriol. 174, 6763-6770 (1992).

37. McCleary, W. R. \& Stock, J. B. Acetyl phosphate and the activation of twocomponent response regulators. J. Biol. Chem. 269, 31567-31572 (1994).

38. Fischer, A. J. \& Lagarias, J. C. Harnessing phytochrome's glowing potential. Proc. Natl Acad. Sci. USA 101, 17334-17339 (2004).

39. Fischer, A. J. et al. Multiple roles of a conserved GAF domain tyrosine residue in Cyanobacterial and plant phytochromes. Biochemistry 44, 15203-15215 (2005).

40. Castillo-Hair, S. M., Fujita, M., Igoshin, O. A. \& Tabor, J. J. An engineered B. subtilis inducible promoter system with over 10,000-fold dynamic range. ACS Synth. Biol. https://doi.org/10.1021/acssynbio.8b00469 (2019)

41. Goodman, D. B., Church, G. M. \& Kosuri, S. Causes and effects of N-terminal codon bias in bacterial genes. Science 342, 475-479 (2013).

42. Bentele, K., Saffert, P., Rauscher, R., Ignatova, Z. \& Blüthgen, N. Efficient translation initiation dictates codon usage at gene start. Mol. Syst. Biol. 9, 675 (2013).

43. Rocha, E. P. C., Danchin, A. \& Viari, A. Translation in Bacillus subtilis: roles and trends of initiation and termination, insights from a genome analysis. Nucleic Acids Res 27, 3567-3576 (1999).

44. Espah Borujeni, A. et al. Precise quantification of translation inhibition by mRNA structures that overlap with the ribosomal footprint in $\mathrm{N}$-terminal coding sequences. Nucleic Acids Res 45, 5437-5448 (2017).

45. Hui, M. P., Foley, P. L. \& Belasco, J. G. Messenger rna degradation in bacterial cells. Annu. Rev. Genet. 48, 537-559 (2014).

46. Guiziou, S. et al. A part toolbox to tune genetic expression in Bacillus subtilis. Nucleic Acids Res 44, 7495-7508 (2016).

47. Radeck, J. et al. The Bacillus BioBrick Box: generation and evaluation of essential genetic building blocks for standardized work with Bacillus subtilis. $J$. Biol. Eng. 7, 29 (2013).

48. Lou, C., Stanton, B., Chen, Y.-J., Munsky, B. \& Voigt, C. A. Ribozyme-based insulator parts buffer synthetic circuits from genetic context. Nat. Biotechnol. 30, $1137-1142$ (2012).

49. Cox, R. S., Surette, M. G. \& Elowitz, M. B. Programming gene expression with combinatorial promoters. Mol. Syst. Biol. 3, 145 (2007).

50. Egbert, R. G. \& Klavins, E. Fine-tuning gene networks using simple sequence repeats. Proc. Natl Acad. Sci. USA 109, 16817-16822 (2012).

51. Farasat, I. et al. Efficient search, mapping, and optimization of multi-protein genetic systems in diverse bacteria. Mol. Syst. Biol. 10, 731 (2014).

52. Iizuka, R., Yamagishi-Shirasaki, M. \& Funatsu, T. Kinetic study of de novo chromophore maturation of fluorescent proteins. Anal. Biochem. 414, 173-178 (2011).

53. Gautier, A. et al. How to control proteins with light in living systems. Nat. Chem. Biol. 10, 533-541 (2014).

54. Ramakrishnan, P. \& Tabor, J. J. Repurposing synechocystis PCC6803 UirS-UirR as a UV-violet/green photoreversible transcriptional regulatory tool in E. coli. ACS Synth. Biol. 5, 733-740 (2016).

55. Chen, X. et al. An extraordinary stringent and sensitive light-switchable gene expression system for bacterial cells. Cell Res. 26, 854-857 (2016).

56. Baumschlager, A., Aoki, S. K. \& Khammash, M. Dynamic blue light-inducible T7 RNA polymerases (Opto-T7RNAPs) for precise spatiotemporal gene expression control. ACS Synth. Biol. 6, 2157-2167 (2017).

57. Ong, N. T., Olson, E. J. \& Tabor, J. J. Engineering an E. coli near-infrared light sensor. ACS Synth. Biol. 7, 240-248 (2018).

58. Frankenberg, N. \& Lagarias, J. C. Phycocyanobilin:ferredoxin oxidoreductase of Anabaena sp. PCC 7120. Biochemical and spectroscopic characterization. J. Biol. Chem. 278, 9219-9226 (2003).

59. Uda, Y. et al. Efficient synthesis of phycocyanobilin in mammalian cells for optogenetic control of cell signaling. Proc. Natl Acad. Sci. USA 114, 11962-11967 (2017).

60. Kyriakakis, P. et al. Biosynthesis of orthogonal molecules using ferredoxin and ferredoxin-NADP+ reductase systems enables genetically encoded PhyB optogenetics. ACS Synth. Biol. (2018). https://doi.org/10.1021/acssynbio.7b00413
61. Castellana, M. et al. Enzyme clustering accelerates processing of intermediates through metabolic channeling. Nat. Biotechnol. 32, 1011-1018 (2014).

62. Wheeldon, I. et al. Substrate channelling as an approach to cascade reactions. Nat. Chem. 8, 299-309 (2016).

63. Liu, Y. \& Montellano, P. R. Ode Reaction intermediates and single turnover rate constants for the oxidation of heme by human heme oxygenase-1. J. Biol. Chem. 275, 5297-5307 (2000).

64. Fujita, M. \& Losick, R. Evidence that entry into sporulation in Bacillus subtilis is governed by a gradual increase in the level and activity of the master regulator Spo0A. Genes Dev. 19, 2236-2244 (2005).

65. Vishnoi, M. et al. Triggering sporulation in Bacillus subtilis with artificial twocomponent systems reveals the importance of proper Spo0A activation dynamics. Mol. Microbiol. 90, 181-194 (2013).

66. Möglich, A., Ayers, R. A. \& Moffat, K. Design and signaling mechanism of light-regulated histidine kinases. J. Mol. Biol. 385, 1433-1444 (2009).

67. Seo, S. W. et al. Predictive design of mRNA translation initiation region to control prokaryotic translation efficiency. Metab. Eng. 15, 67-74 (2013).

68. Helmann, J. D. Compilation and analysus of Bacillus subtilis $\sigma \mathrm{A}$-dependent promoter sequences: evidence for extended contact between RNA polymerse and upstream promoter DNA. Nucleic Acids Res 23, 2351-2360 (1995).

69. Jarmer, H. et al. Sigma A recognition sites in the Bacillus subtilis genome. Microbiology 147, 2417-2424 (2001).

70. Voskuil, M. I. \& Chambliss, G. H. The -16 region of Bacillus subtilis and other gram-positive bacterial promoters. Nucleic Acids Res 26, 3584-3590 (1998).

71. Raab, D., Graf, M., Notka, F., Schödl, T. \& Wagner, R. The GeneOptimizer Algorithm: using a sliding window approach to cope with the vast sequence space in multiparameter DNA sequence optimization. Syst. Synth. Biol. 4, 215-225 (2010).

72. Fujita, M. \& Losick, R. An investigation into the compartmentalization of the sporulation transcription factor $\sigma \mathrm{E}$ in Bacillus subtilis. Mol. Microbiol. 43, 27-38 (2002).

73. Landry, B. P., Palanki, R., Dyulgyarov, N., Hartsough, L. A. \& Tabor, J. J. Phosphatase activity tunes two-component system sensor detection threshold. Nat. Commun. 9, 1433 (2018).

74. Engler, C., Gruetzner, R., Kandzia, R. \& Marillonnet, S. Golden gate shuffling: a one-pot dna shuffling method based on type IIs restriction enzymes. PLoS ONE 4, e5553 (2009).

75. Zadeh, J. N. et al. NUPACK: analysis and design of nucleic acid systems. J. Comput. Chem. 32, 170-173 (2011).

76. Extraction of PCB from Spirulina - OpenWetWare. Available at: https:// openwetware.org/wiki/Extraction_of_PCB_from_Spirulina. (Accessed: 2nd October 2018)

77. Gerhardt, K. P. et al. An open-hardware platform for optogenetics and photobiology. Sci. Rep. 6, 35363 (2016).

78. Castillo-Hair, S. M. et al. FlowCal: A user-friendly, open source software too for automatically converting flow cytometry data from arbitrary to calibrated units. ACS Synth. Biol. 5, 774-780 (2016).

79. Newville, M. et al. Lmfit: Non-linear least-square minimization and curvefitting for Python. Astrophys. Source Code Libr. 2016ascl.soft06014N (2016).

80. Castillo-Hair, S. M., Baerman, E. A., Fujita, M., Igoshin, O. A. \& Tabor, J. J. Data from 'Optogenetic control of Bacillus subtilis gene expression'. Figshare https://doi.org/10.6084/m9.figshare.8198999

81. Kim, L., Mogk, A. \& Schumann, W. A xylose-inducible Bacillus subtilis integration vector and its application. Gene 181, 71-76 (1996).

82. Rygus, T., Scheler, A., Allmansberger, R. \& Hillen, W. Molecular cloning, structure, promoters and regulatory elements for transcription of the Bacillus megaterium encoded regulon for xylose utilization. Arch. Microbiol. 155, 535-542 (1991).

83. Dahl, M. K., Degenkolb, J. \& Hillen, W. Transcription of the xyl operon is controlled in Bacillus subtilis by tandem overlapping operators Spaced by four Base-pairs. J. Mol. Biol. 243, 413-424 (1994).

84. Chen, Y.-J. et al. Characterization of 582 natural and synthetic terminators and quantification of their design constraints. Nat. Methods 10, 659-664 (2013).

85. Quisel, J. D., Burkholder, W. F. \& Grossman, A. D. In vivo effects of sporulation kinases on mutant spo0a proteins in Bacillus subtilis. J. Bacteriol. 183, 6573-6578 (2001).

\section{Acknowledgements}

We thank Karl Gerhardt for performing the PCB purification protocol. This work was supported by a Michel Systems Biology Innovation Award from Rice University, the National Institutes of Health (1R21AI115014-01A1), and the National Science Foundation (DMR 1611044). O.A.I. also acknowledges support from the National Science Foundation (MCB-1616755) and the Welch Foundation (Grant C-1995). 


\section{Author contributions}

S.M.C., M.F., O.A.I., and J.J.T. conceived of the project. S.M.C. designed and built DNA constructs. S.M.C. and E.A.B. collected data. S.M.C. performed all data analyses. O.A.I. and J.J.T. supervised the project. S.M.C., M.F., O.A.I., and J.J.T. wrote the manuscript.

\section{Additional information}

Supplementary Information accompanies this paper at https://doi.org/10.1038/s41467019-10906-6

Competing interests: The authors declare no competing interests.

Reprints and permission information is available online at http://npg.nature.com/ reprintsandpermissions/

Peer review information: Nature Communications thanks Mary Dunlop and other anonymous reviewer(s) for their contribution to the peer review of this work. Peer reviewer reports are available.
Publisher's note: Springer Nature remains neutral with regard to jurisdictional claims in published maps and institutional affiliations.

(c) (i) Open Access This article is licensed under a Creative Commons Attribution 4.0 International License, which permits use, sharing, adaptation, distribution and reproduction in any medium or format, as long as you give appropriate credit to the original author(s) and the source, provide a link to the Creative Commons license, and indicate if changes were made. The images or other third party material in this article are included in the article's Creative Commons license, unless indicated otherwise in a credit line to the material. If material is not included in the article's Creative Commons license and your intended use is not permitted by statutory regulation or exceeds the permitted use, you will need to obtain permission directly from the copyright holder. To view a copy of this license, visit http://creativecommons.org/ licenses/by/4.0/.

(c) The Author(s) 2019 